\title{
Liberté religieuse contre liberté d'expression? Pressions de conformité et rhétorique politiquement correcte
}

Guy Haarscher

\section{CpenEdition}

\section{Journals}

Édition électronique

URL : https://journals.openedition.org/rdr/1211

DOI : 10.4000/rdr.1211

ISSN : 2534-7462

Éditeur

Presses universitaires de Strasbourg

\section{Édition imprimée}

Date de publication : 16 novembre 2020

Pagination : 33-53

ISBN : 979-10-344-0073-7

ISSN : 2493-8637

\section{Référence électronique}

Guy Haarscher, «Liberté religieuse contre liberté d'expression? Pressions de conformité et rhétorique politiquement correcte », Revue du droit des religions [En ligne], 10 | 2020, mis en ligne le 16 novembre 2020, consulté le 02 mai 2022. URL : http://journals.openedition.org/rdr/1211 ; DOI : https://doi.org/ $10.4000 /$ rdr. 1211 


\section{LIBERTÉRELIGIEUSE CONTRE LIBERTÉ D'EXPRESSION? PRESSIONSDECONFORMITÉ ETRHÉTORIQUE POLITIQUEMENT CORRECTE}

\section{GUy HAARSCHER}

Professeur émérite de l'Université Libre de Bruxelles (ULB)

\section{RÉSUMÉ}

Les dangers que court la liberté d'expression ne sont pas toujours liés à des attaques frontales, comme l'est l'accusation de blasphème. De plus en plus souvent, la rhétorique liberticide se présente comme une illustration de la défense des droits de l'homme. Ainsi la liberté religieuse, le refus du discours de haine et le droit à la réputation (corrélatif de la notion de diffamation) - pour ne citer que ces trois notions - peuvent-ils servir à une mise en danger de la liberté d'expression. Péril quasi invisible pour qui ne critique pas sérieusement les concepts utilisés, puisque le «vilain mot» de blasphème n'est plus prononcé et que des concepts très politiquement corrects viennent à sa place jouer le rôle de «censeurs » de l'expression libre. Je voudrais dans le présent article fournir quelques éléments de déconstruction de cette rhétorique périlleuse pour les libertés.

\section{ABstract}

The dangers to freedom of expression are not always linked to frontal attacks, as is the charge of blasphemy. The freedom-destroying rhetoric increasingly takes on the appearance of the defence of human rights. Thus, religious freedom, the rejection of hate speech and the right to reputation (a correlative of the concept of defamation) - to name but three notions - can serve to endanger freedom of expression. This is almost invisible for those who do not seriously criticize the concepts used, since the "ugly word" of blasphemy is no longer pronounced and very politically correct concepts come to play the role of "censors" of free expression in its place. In this article I would like to provide some elements of deconstruction of this perilous rhetoric for freedoms. 


\section{INTRODUCTION}

Il est loin, du moins dans nos démocraties, le temps où les «injures» adressées à Dieu ou à la religion se voyaient sanctionnées comme telles - comme des blasphèmes. La fin du $\mathrm{xx}^{\mathrm{e}}$ siècle a témoigné à cet égard d'un progrès et d'une régression, les deux se trouvant liés. Progrès: on ne protégerait plus la religion, mais plutôt les individus et leurs droits. Régression : et si la vieille répression du blasphème se poursuivait toujours - certes atténuée dans ses effets, grâce au progrès de la justice pénale et à une plus grande «douceur des peines ${ }^{1} »-$ mais sous le masque de la protection des droits de l'homme? Ce qu'on aurait gagné se payerait par une défaite de la pensée.

Je me propose, dans les limites de ce bref article, de mettre à jour différentes stratégies rhétoriques par lesquelles une telle idée se trouve mise en œuvre.

Mais tout d'abord, je voudrais relier la question du blasphème à celle de la «politique des identités ${ }^{2} »$. Quand une religion est majoritaire, quand elle se trouve inscrite dans une longue tradition, quand elle définit, comme le dit Max Weber, «l'autorité de l'"éternel hier" ${ }^{3}$ ", l'individu adhère en général de façon normale et sous une contrainte sociale plus ou moins ressentie, à la croyance, à la confession dominante. Il ne se pose pas vraiment la question de son identité religieuse, dans la mesure où elle va en quelque sorte de soi.

Dans le monde contemporain, les progrès de la liberté religieuse, et plus largement de la liberté de conscience (on a aussi le droit de ne pas croire), ont fait se multiplier les orientations, interprétations, sensibilités: si l'homogénéité n'est pas imposée d'en haut, si chacun est libre de vivre ses engagements spirituels à condition de respecter la loi, le pluralisme s'imposera de lui-même. De plus, les mouvements migratoires et les modifications de frontières (en particulier en Europe centrale et orientale après la première guerre mondiale) ont créé des groupes minoritaires, pratiquant parfois des religions aux valeurs et pratiques éloignées de celles de la majorité. Cette dernière s'est à son tour définie de façon identitaire, poussée (souvent par ses extrêmes) à se demander «qui » elle était et quelles concessions elle devait accepter pour vivre avec des communautés minoritaires « différentes».

1. M. Foucault, Surveiller et punir, Paris, Gallimard, 1975, p. 106-134.

2. V. F. Funuyama, Identity. Contemporary identity politics and the struggle for recognition, London, Profile Books, 2018.

3. M. Weber, Le savant et le politique [conférences de 1919], Paris, Plon, 1959, p. 126. 
Ce raidissement identitaire fonctionnant pour ainsi dire en miroir a créé les conditions du radicalisme minoritaire (souvent islamiste) opposé à un radicalisme populiste, «blanc», majoritaire, parfois défini comme «chrétien», ce qui pourrait malheureusement mener à un conflit des «civilisations» au sens de Huntington ${ }^{4}$.

Mais si l'on définit, loin des tentations populistes identitaires, les règles du jeu à accepter - notre «mode de vie», dit-on aujourd'hui de façon d'ailleurs controversée - comme le respect des droits de l'homme, la tolérance, l'égalité entre les femmes et les hommes, les droits des homosexuels, la liberté de la science, et surtout la liberté d'expression, alors les revendications identitaires majoritaires (pouvant virer au populisme) ou minoritaires (risquant d'engendrer de la violence, voire du terrorisme) seront peut-être circonscrites.

Mais en même temps, les crispations identitaires à coloration religieuse sont, malgré les différences affichées, souvent curieusement similaires: refus de la science qui désenchante le monde religieux, refus d'accorder aux femmes un statut égal à celui des hommes, intolérance à la critique, refus de l'avortement, des droits des homosexuels, et de la liberté d'expression quand elle concerne la critique, parfois satirique et vigoureuse, des religions ou d'une religion particulière. Les positions minoritaires $\mathrm{du} \mathrm{XxI}^{\mathrm{e}}$ siècle ressemblent parfois très fort à celles du $\mathrm{XIX}^{\mathrm{e}}$ siècle résistant au grand mouvement de laïcisation et de sécularisation.

J'essaie de montrer dans le présent article que la protection des «identités » ne peut réussir qu'en défendant de façon rigoureuse et cohérente les valeurs du vivre ensemble et la liberté de la pensée. Il faut se montrer capable de déceler derrière des demandes "politiquement correctes» la vieille incapacité de certains à accepter la subordination, en cas de conflit, de la loi de Dieu à celle du demos, surtout quand ce dernier s'incarne dans des systèmes constitutionnels garantissant la liberté religieuse.

\section{LA QUESTION DES SENSIBILITÉS RELIGIEUSES}

Une stratégie couramment utilisée (elle l'est notamment par la Cour européenne des droits de l'homme, qui a réaffirmé sa position de 1994 dans un arrêt récent de $2018^{5}$ ) consiste à faire glisser la problématique, si l'on peut

4. S. P. Huntington, Le choc des civilisations, Paris, Odile Jacob, 2000.

5. CEDH, 20 sept. 1994, no 3470/87, Otto-Preminger-Institut c. Autriche; 25 oct. 2018, $\mathrm{n}^{\circ} 38450 / 12$, E.S. c. Autriche. V. G. HAARSCHER, «Le blasphémateur sous les fourches caudines des juges de Strasbourg», RTDH 2019, p. 505. 
dire, de Dieu au croyant, du Créateur à la créature. Dans ce cas, on ne soutiendra plus directement que l'expression est «blasphématoire», mais qu'elle blesse les sensibilités des croyants: de telles horreurs proférées à l'égard de ce qui leur est spirituellement le plus cher les empêcheraient de pratiquer paisiblement leur culte. Si on donne raison à ces derniers, on devra respecter leur «droit de l'homme » (en l'occurrence la liberté religieuse) en limitant cet autre droit de l'homme que constitue la liberté d'expression. Mais comment choisir entre les deux? Le simple fait de poser la question en ces termes indique que l'on a changé d'univers intellectuel.

Dans la problématique traditionnelle (toujours d'actualité dans certains pays comme le Pakistan par exemple), le blasphème mettait en présence un être humain tenant un certain type de «discours» et Dieu ou ses représentants officiels (les Églises au sens large du terme, soutenues par les États). La situation était intellectuellement claire: un droit de l'homme (le droit à la liberté d'expression) se trouvait opposé à une religion officielle (ou ce qui en subsiste dans les pays qui ont conservé des lois anti-blasphème). En revanche, dans la formulation rhétorique contemporaine, deux droits de l'homme se font face: le conflit s'est transformé en un conflit dit «systémique» (au sein même du «système» des droits de l'homme). Une telle stratégie permet de ne pas mettre en cause le primat, reconnu par les grandes démocraties libérales, des droits de l'homme sur les autres normes, en particulier celles supposées d'inspiration divine, et donc à caractère sacré pour une partie de la population dans les sociétés pluralistes qui sont les nôtres. Mais évidemment, que le discours soit interdit parce qu'il est jugé blasphématoire ou parce qu'il offense les sensibilités des croyants ne change pas grand-chose quant au résultat de l'opération: ce que l'on ne saurait voir ou entendre ne sera pas montré ou proféré.

Mais si deux droits fondamentaux (liberté d'expression et liberté religieuse) sont mis sur le même plan - ils ne sont d'habitude pas hiérarchisés, et ne le sont en tout cas pas dans la Convention européenne des droits de l'homme -, comment trancher entre ces deux prétentions? On pourrait imaginer que la liberté religieuse l'emporte totalement, dans une société où tout propos jugé offensant par tout croyant se verrait interdit. Une telle situation est évidemment impensable dans une démocratie libérale et pluraliste: un tel «politiquement correct» poussé à l'absolu et à l'absurde aboutirait pour le coup à un «effet de gel» (ce que l'on appelle aux États-Unis chilling effect ${ }^{6}$ ) du discours.

6. V. Cour suprême des États-Unis, Lamont v. Postmaster General, 381 U.S. 301 (1965). 
Les systèmes juridiques des démocraties libérales ont donc dû s'accommoder d'exagérations, comme en témoignent ces deux citations célèbres:

«Dans le champ de la foi religieuse et dans celui des opinions politiques, des différences marquées apparaissent. Dans ces deux domaines, les principes d'un individu peuvent apparaître comme l'erreur la plus flagrante aux yeux de son voisin. Pour persuader les autres de la validité de son propre point de vue, le plaideur a parfois recours à l'exagération; il peut calomnier des hommes qui ont occupé, ou occupent encore, une position importante dans une communauté religieuse ou dans l'État; il peut même lui arriver d'énoncer des contre-vérités. Mais les membres de cette nation ont considéré, à la lumière de l'histoire, qu'en dépit de la probabilité d'excès et d'abus, ces libertés sont, pour une vision à long terme, essentielles à l'opinion éclairée et à la bonne conduite des citoyens d'une démocratie ${ }^{7}$.»

«Son rôle de surveillance commande à la Cour de prêter une extrême attention aux principes propres à une "société démocratique". La liberté d'expression constitue l'un des fondements essentiels de pareille société, l'une des conditions primordiales de son progrès et de l'épanouissement de chacun. Sous réserve du paragraphe 2 de l'article 10 (art. 10-2), elle vaut non seulement pour les "informations" ou "idées" accueillies avec faveur ou considérées comme inoffensives ou indifférentes, mais aussi pour celles qui heurtent, choquent ou inquiètent l'État ou une fraction quelconque de la population. Ainsi le veulent le pluralisme, la tolérance et l'esprit d'ouverture sans lesquels il n'est pas de "société démocratique"8."

Mais dans de telles conditions, étant donné l'exigence de mise en balance de deux droits d'égale importance ${ }^{9}$, le défenseur des sensibilités religieuses ne sera-t-il pas nécessairement défait? Si l'exercice de la liberté d'expression est considéré comme légitime jusqu'à heurter, choquer ou inquiéter, ou à se livrer à des «excès et abus» probables, le blasphème tel que traduit dans le langage des droits de l'homme aura toutes les chances d'être permis ${ }^{10}$.

7. Cantwell v. Connecticut, 310 U.S. 296 (1940), cité par le très libéral juge Brennan dans l'opinion majoritaire de l'arrêt New York Times v. Sullivan, 376 U.S. 254 (1964) [traduit et souligné par nous]. Brennan, qui siégea de 1956 à 1990, se joignit à l'opinion majoritaire dans Hustler Magazine, Inc. v. Falwell, 485 U.S. 46 (1988). V. infra.

8. CEDH, 7 déc. 1976, no 5493/72, Handyside c. Royaume-Uni, \& 49 [souligné par nous].

9. Aux États-Unis, la liberté d'expression et la liberté religieuse occupent toutes deux une preferred position, selon l'arrêt de la Cour suprême Murdock v. Pennsylvania, 319 U.S. 105 (1943), ce qui ne tranche pas la question.

10. C'est le cas par la Cour suprême des États-Unis, dans l'arrêt Joseph Burstyn, Inc. v. Wilson, 343 U.S. 495 (1952). 
On voit donc déjà se profiler deux objections à la transposition de la problématique du blasphème (qui oppose un État liberticide à celui qui exerce ou tente d'exercer sa liberté d'expression) dans le domaine des droits de l'homme: argumenter en invoquant les sensibilités religieuses se révèle moins facile que l'attaque frontale, à savoir le refus des discours «insultants » adressés à Dieu et aux personnages des grands récits sacrés (voire aux dignitaires des Églises). D’une part, il serait possible de rejeter d'emblée l'opération rhétorique comme étant sophistique, en déclarant (ce qu'ont fait des juges dissidents dans l'affaire Otto-Preminger-Institut c. Autriche ${ }^{11}$ ) que la liberté religieuse n'inclut pas le droit de ne pas être heurté, choqué, inquiété dans ses «sensibilités». On pourrait simplement affirmer que, dans une société pluraliste où la vérité de l'un peut toujours être considérée comme un blasphème pour l'autre, les individus, s'ils ne vivent pas sous cloche (ce n'est pas démocratiquement souhaitable), doivent s'accommoder de discours parfois dérangeants pour eux, sauf cas de harcèlement direct: par exemple en 1989, les Versets sataniques de Salman Rushdie ont été virulemment dénoncés et son auteur menacé de mort, jusqu'à faire l'objet d'une fatwa de la part de l'imam Khomeiny, à l'époque « Guide de la Révolution de la République islamique d'Iran ». Or il s'agissait en l'occurrence d'un livre que personne n'était obligé d'acheter ou de lire. Si - hypothèse absurde au demeurant - Rushdie était allé placarder les Versets sataniques dans les mosquées ou dans leurs alentours de façon à harceler les musulmans et à les forcer de voir ce qu'ils ne voulaient pas voir, le bon sens aurait exigé qu'un tel usage outrancier du droit à la liberté d'expression soit interdit.

Cette seconde objection nous mène à une distinction terminologique capitale entre deux sortes d'espaces publics. Je parle ici d'espaces ouverts au public et non des pouvoirs publics ou de l'État au sens large du terme. Il y a d'une part un public captif, d'autre part un public volontaire. Pour poursuivre l'argument à partir de l'exemple que je viens de donner, les musulmans qui ne pourraient pas ne pas voir les phrases choquantes des Versets placardées partout sur leur passage (on pourrait imaginer qu'elles soient écrites sur les murs des couloirs du métro) constitueraient le paradigme même du public captif. Dans ce cas on pourrait accepter que l'expression soit moins protégée, dans la mesure où elle risquerait effectivement de choquer des individus passant en un lieu (et parfois obligés de le faire, par exemple si la station de métro constitue une étape obligée vers leur lieu de travail) et voyant ce qu'ils n'étaient sûrement pas venus pour voir.

11. Otto-Preminger-Institut c. Autriche, précit., opinion dissidente des juges Palm, Pekkanen et Makarczyk, \& 6. 
Il en va différemment pour un public que j'appellerai «volontaire», par exemple dans une salle de cinéma ou de théâtre (ou lors de la lecture d'un livre): ce public sait ce qu'il veut voir et a accompli un acte de volonté précis: payer un ticket et entrer dans une salle, acheter et ouvrir un ouvrage, etc. S'il est choqué, c'est soit parce qu'il le voulait, soit parce que le contenu du spectacle ou du livre était plus «hard» qu'il ne l'avait cru avant d'entamer l'expérience de spectateur, d'auditeur, de lecteur, etc.

Mais justement: son expérience diffère radicalement de mon exemple précédent: le membre de cette entité vague que l'on nomme «public captif » n'est absolument pas là (à la mosquée, dans le métro...) pour voir ou lire quelque chose: il vise un autre but (aller prier, se rendre à son travail...). On peut comprendre qu'il n'aime vraiment pas être confronté à ces discours (sensu lato) qui le choquent et qui sont, c'est le moins que l'on puisse dire, non sollicités par lui. À l'opposé, le public volontaire veut lire, écouter ou voir quelque chose, ce qui est parfaitement son droit - et si, malgré tout, ce qu'il découvre le heurte, il n'a qu'à s'en prendre à lui-même, sortir de la salle ou refermer le livre incriminé.

Cette dernière considération me mène à une remarque importante. Si vous achetez un livre ou allez voir un film ou une pièce, c'est parce que vous en avez été averti - sinon vous n'en connaîtriez même pas l'existence. Les espaces publics captif et volontaire ne sont pas totalement isolés l'un de l'autre. Il faut bien en effet que la publicité fasse connaître l'existence d'une ouvre (spectacle, parution d'un livre, etc.) pour que cette dernière, justement, trouve son public (volontaire). La publicité se situe à l'interface des deux espaces, elle informe les «clients» potentiels et fait la promotion du produit culturel en question. Or cette promotion se fait nécessairement au sein du public captif: les publicités «s'invitent», sollicitent l'attention en étant placées en des points stratégiques de façon à être vues par ceux qui passent (ou regardent la télévision, particulièrement en prime time). D'où la difficulté, évidente au demeurant: si ce que montre la pub se révèle aussi choquant que le contenu de l'œuvre réservé à un public volontaire, la différence entre les deux espaces perdra tout sens. La publicité se doit donc d'être plus «prudente», moins radicale dans l'expression que ce qu'elle annonce et à partir de quoi le public captif qui sera «tombé dessus » (à moins qu'elle ne «vienne à lui» quand il regarde un film à la télévision ou consulte un site Internet) décidera en citoyen libre d'y aller ou de ne pas y aller, d'acheter le livre ou de n'en rien faire.

On se rappelle l'affaire suscitée par la publicité du film de Milos Forman, The People v. Larry Flynt, en 1997. Personnage sulfureux, Larry Flynt, 
fondateur et directeur d'une revue pornographique (Hustler Magazine), avait publié une satire du télévangéliste Jerry Falwell. La réaction indignée de ce dernier suscita une affaire judiciaire qui suivit son chemin jusqu'à la Cour suprême des États-Unis ${ }^{12}$. Ce qui nous intéresse ici, c'est le film que Forman a tiré de toute l'histoire, et en particulier, la façon dont il en a imaginé la publicité ${ }^{13}$. Cette dernière a suscité sa propre affaire en France. Elle présentait Larry Flynt, tout petit, vêtu seulement d'un lange aux couleurs du drapeau américain, et en position de crucifié sur le pubis d'une jeune femme en bikini. Le lange-drapeau rappelait aux connaisseurs que Flynt s'était présenté un jour dans cet appareil (presque le plus «simple») devant le juge d'un tribunal américain et qu'il avait été condamné pour profanation de l'emblème des États-Unis en 1983.

Mais ce n'est pas cet accoutrement qui choqua une partie du public «captif» passant dans les rues de Paris ou d'une autre grande ville pour vaquer à ses occupations quotidiennes. Ce fut l'imitation de la position du Christ. Le publicitaire (ou Forman lui-même) avait sans doute voulu symboliser par cette image la liberté d'expression «crucifiée» par la bigoterie des ligues de vertu et des télévangélistes américains. Mais en France, des intégristes et une association liée à l'extrême droite tentèrent de faire interdire la publicité en cause parce qu'elle heurtait prétendument les sensibilités religieuses des catholiques. Ils furent déboutés par un juge qui affirma notamment que, dans le fond, la position de Larry Flynt sur l'image ne ressemblait pas tellement à Jésus sur la Croix. Certains auraient sans doute été plus rassurés si le magistrat avait considéré qu'il y avait ressemblance et que l'expression était protégée. Quoi que l'on pense à ce sujet, il est incontestable que la publicité était assez «limite» et qu'elle pouvait choquer un croyant conservateur obligé de passer en ces lieux et donc devenu "public captif».

On voit donc la difficulté posée par la question de l'interface publicitaire entre les espaces captif et volontaire. Elle n'est pas insurmontable - toute théorie possède ses hard cases. Mais elle montre évidemment par comparaison ce qu'aurait d'absurde une censure d'expressions auxquelles ne serait confronté qu'un public volontaire et qui, par définition ne pourraient choquer ceux qui auraient décidé, sur la base de la publicité ou du ouï-dire, de ne pas faire l'expérience - à moins qu'ils n'en aient tout simplement pas entendu parler. C'est sur ce point précis que se révèle la fragilité intrinsèque du processus de traduction que j'ai brièvement décrit plus haut: quand on

12. Hustler Magazine, Inc. v. Falwell, 485 U.S. 46 (1988).

13. V. pour ce qui suit «L'affiche et les principes», Le Monde, 20 févr. 1997. 
parle de blasphème, on vise une offense à Dieu ou à des personnages sacrés; c'est une affaire entre le blasphémateur et la divinité, duo auquel viennent se joindre les censeurs supposés parler au nom de Dieu. On a souvent défini le blasphème comme un victimless crime, un crime sans victime humaine, seule l'entité transcendante se trouvant attaquée.

On a tellement pris l'habitude de parler du blasphème dans des termes modernes (comme le fait de heurter les sensibilités des croyants) que l'on a oublié les implications classiques - insoutenables à notre regard mais parfaitement logiques - de la notion en tant que telle. L'offense à Dieu ne dépend en principe pas du contexte dans lequel elle a lieu: dans la sphère privée, dans l'espace public captif ou dans l'espace public volontaire. Une attaque contre Dieu est inacceptable où qu'elle se produise et quel qu'en soit l'auteur. Si des croyants sont présents dans le public et ne se plaignent pas - ne sont pas heurtés, choqués, inquiétés -, c'est, diront les gardiens de la foi, parce qu'ils sont trop tièdes, parce qu'ils ne sont pas suffisamment prêts à défendre leur Dieu et leur foi contre les attaques des mécréants ou des hérétiques. Ces croyants seront taxés de «tolérantisme»-ils auront toléré, accepté, même sans "aimer», ce qu'ils n'auraient jamais dû laisser advenir. Le concept de tolérantisme indique bien qu'avant d'être considérée comme une vertu, la tolérance fut stigmatisée comme un vice grave ${ }^{14}$.

Ce monde du blasphème appréhendé tel quel, sans faux-fuyant ni tentative de «modernisation», possède au moins le mérite de la simplicité. C'est une attaque contre Dieu, les croyants ne se trouvant qu'indirectement concernés: ils pourraient bien sûr être choqués, mais même s'ils ne l'étaient pas (ce dont les accuseraient les censeurs rigoristes), ou s'il n'y avait pas de croyant dans le public, le crime contre Dieu subsisterait.

Or justement, quand on opère le transfert de Dieu aux croyants, quand on soutient que, «non, ce n'est pas Dieu, notre Dieu qui est en cause dans notre demande de censure, mais le croyant choqué», encore faudrait-il que ce dernier se manifeste - encore devrait-il exister une victime et qu'il ne s'agisse pas seulement d'un victimless crime!

Il est assez absurde de considérer que le simple fait qu'existe une expression litigieuse puisse heurter les sensibilités des croyants alors qu'elle apparaît en un lieu où ces derniers ne se trouvent pas (parce qu'ils n'ont pas accompli l'acte de volonté nécessaire). En quoi des croyants peuvent-ils être affectés

14. P. Thierry, La tolérance, société démocratique, opinions, vices et vertus, Paris, PUF, 1997, p. 6 et $s$. 
dans leur exercice du droit à la liberté religieuse par des expressions qu'ils peuvent éviter si - ce qui est évidemment parfaitement leur droit - ils les trouvent choquantes pour leurs convictions? Si la publicité (par opposition à la «provocation» de Forman) se révèle prudente, réservée, informative, certes accrocheuse (c'est son but) mais sans choquer - si donc cette petite partie de l'espace public captif n'est pas par elle-même choquante -, il semble particulièrement déraisonnable de censurer l'expression, sauf à assumer la conception pure et dure du blasphème, injure adressée à Dieu, fût-ce au fond d'une salle obscure dans laquelle ne se sont assis que ceux qui ont décidé d'y être.

Bien entendu, celui qui décide librement de se confronter à l'expression litigieuse et s'en trouve choqué bénéficie dans ce cas lui aussi de toutes les possibilités associées au droit à la liberté d'expression: il peut réagir, déclarer que la pièce, le film, le livre est nul, écrire un article démolissant l'œuvre, etc. Mais il n'est pas raisonnable de censurer une expression à laquelle personne n'est obligé d'être confronté. On préférera donc l'action que je viens de mentionner (un citoyen réagissant en rédigeant un compte rendu dévastateur) à la demande d'interdiction. Comme l'a dit le grand juge Brandeis, qui siégea à la Cour suprême des États-Unis de 1916 à 1939, il vaut mieux plus de discours (à l'initiative de celui qui exerce sa liberté d'expression contre une expression qu'il conteste) que moins de discours (à l'initiative de celui qui prétend brandir sa liberté religieuse contre la liberté d'expression):

«S'il reste du temps pour exposer à travers la discussion les erreurs et sophismes, pour éviter le mal par les processus d'éducation, le remède à appliquer est un supplément de discours, et non le silence forcé. Seule une urgence peut justifier la répression ${ }^{15}$.»

Mais nombreux sont ceux qui ne tiennent pas compte de l'objection du public captif - pourtant dirimante. À supposer qu'on la mette donc de côté, une difficulté subsiste si, à l'instar de la Cour européenne des droits de l'homme dans son arrêt Handyside c. Royaume-Uni de 1976, on considère que les propos qui heurtent, choquent ou inquiètent doivent être protégés. On suppose donc dans cette hypothèse qu'un croyant est heurté dans sa sensibilité religieuse (ce qui, rappelons-le, apparaît hautement improbable si on se trouve dans le cas d'un public volontaire). Il invoquera son droit de pratiquer paisiblement sa religion, ce qui lui serait impossible si «existe»

15. Whitney v. California, 274 U.S. 357 (1927), opinion concordante du juge Brandeis [souligné par nous]. 
quelque part un discours au caractère si dénigrant. L'auteur des propos «blasphématoires» invoquera l'obiter dictum de l'arrêt Handyside, reconnaîtra le caractère choquant de ses propos, mais rappellera que c'est permis, le fait que son contradicteur aime ou pas leur contenu n'entrant pas en ligne de compte.

Il serait alors possible - mais on verserait dans la sophistique pure et simple - de mettre en cause des propos qui heurteraient trop, qui iraient vraiment trop loin, qui se révéleraient offensants sans nécessité - qui blesseraient gratuitement. La thèse serait dès lors la suivante: on a le droit de tenir des propos qui heurtent, choquent ou inquiètent les croyants tant que ces « exagérations» servent un débat d'intérêt public (l'impertinence est parfois nécessaire face à la pression dogmatique de conformité). Si l'outrance va au-delà, si les propos deviennent gratuitement offensants, l'expression ne devrait plus être permise ${ }^{16}$.

Mais une telle position créerait inévitablement un fort sentiment d'insécurité juridique. Comment savoir à l'avance si un livre, une pièce, un film seront considérés par un juge comme «simplement» choquants (permis), ou bien gratuitement offensants (interdits) ${ }^{17}$ ?

Pour en arriver à une telle conclusion (la censure des offenses « gratuites»), il aura fallu franchir des étapes périlleuses et recourir à des arguments dont la plupart sont carrément spécieux. Il aura fallu traduire la problématique du blasphème (liberté d'expression contre État défenseur de la religion) en conflit systémique entre droits de l'homme (liberté d'expression contre liberté de religion), mettre sous le boisseau la différence cruciale entre public captif et public volontaire, inventer une distinction génératrice d'insécurité juridique entre propos qui choquent et propos qui offensent sans nécessité, tout cela sans qu'il ne s'agisse en aucune manière d'attaques contre les personnes, lesquelles ne peuvent se sentir concernées qu'indirectement, pour ainsi dire par rebond, quand des idées contredisent fortement leurs convictions. Cette tentative de rendre compatible la répression du blasphème en le relookant, en attaquant la liberté d'expression au nom d'un autre droit de l'homme, ne peut que fragiliser dangereusement cette dernière.

16. C'est le raisonnement qu'on trouve dans l'arrêt Otto-Preminger-Institut c. Autriche, précit., $\S 49$, et encore tout récemment dans l'arrêt E.S. c. Autriche, précit., § 43. V. G. HaARSCHER, «Le blasphémateur sous les fourches caudines des juges de Strasbourg», art. cit.

17. V. les arrêts de la Cour européenne des droits de l'homme cités ci-dessus, ainsi que d'autres arrêts analysés dans G. HAARSCHER, Comme un loup dans la bergerie. Les libertés d'expression et de pensée au péril du politiquement correct, Paris, Cerf, 2016, p. 49-78. 
La référence aux propos gratuitement offensants ne mène certes pas nécessairement à des décisions attentatoires à la liberté d'expression, mais on peut dire qu'elle suspend pour ainsi dire au-dessus de cette dernière une sorte d'épée de Damoclès.

La Cour a rendu des arrêts en faveur de la liberté d'expression dans plusieurs affaires liées au blasphème.

On se souvient par exemple d'une affaire dans laquelle un journaliste slovaque avait vivement attaqué l'archevêque Sokol, parce que ce dernier avait demandé le retrait du film Larry Flynt de Milos Forman (ainsi que de l'affiche publicitaire) dont nous avons parlé plus haut. L'archevêque avait notamment été traité d'« ogre» et les catholiques avaient été incités à quitter leur Église. La Cour avait considéré que la condamnation de Klein (le journaliste) constituait une violation de l'article 10 de la Convention ${ }^{18}$.

Plus récemment, en Lituanie ${ }^{19}$, un publicitaire avait été condamné pour avoir représenté Jésus et Marie tatoués dans le cadre de la promotion d'une marque de vêtements. Au § 77 de l'arrêt, la Cour affirmait que les publicités n'étaient pas gratuitement offensantes (ni d'ailleurs n'incitaient à la haine). Elle déclarait finalement que la Lituanie avait violé la Convention en son article 10 .

La même année, la Cour rendait un arrêt dans la célèbre affaire des Pussy Riots, condamnées en Russie pour avoir tenté de faire une "performance» provocatrice dans une église de Moscou ${ }^{20}$. La manière dont les jeunes femmes avaient été traitées entraîna la condamnation de la Russie par la Cour: l'État russe avait violé plusieurs articles de la Convention, dont l'article 10.

Dans ces trois cas, l'«épée de Damoclès» ne s'est pas abattue sur les requérants, mais, au moins dans les deux premiers, la Cour a mentionné la gratuité des propos offensants comme cause légitime de limitation de la liberté d'expression, même si elle a considéré que cette notion ne s'appliquait pas dans les affaires considérées.

Nous venons de voir que l'argument du respect des sensibilités peut (certes de façon trop hâtive et superficielle) recevoir l'adhésion de défenseurs de la démocratie libérale: n'est-il pas question de respect des sensibilités, de droits des individus offensés? Nous avons vu qu'en réalité cet argument servait

18. CEDH, 31 oct. 2006, n $72208 / 01$, Klein c. Slovaquie.

19. CEDH, 30 janv. 2018, no 69317/14, Sekmadienis Ltd. c. Lituanie.

20. CEDH, 17 juill. 2018, $\mathrm{n}^{\circ}$ 38004/12, Mariya Alekhina et a. c. Russie. 
à reconduire la problématique de la répression du blasphème par d'autres moyens. Mais il est d'autres manières de «droit-de-l'hommiser» le blasphème.

\section{LA DIFFAMATION (COLLECTIVE)}

Une limite considérée en général comme légitime dans les sociétés de démocratie libérale est constituée par la réputation d'autrui: il est interdit de diffamer les personnes, de porter atteinte à leur réputation en répandant des mensonges pour leur nuire. Et effectivement, la notion de diffamation a souvent été utilisée (y compris jusqu'à l'absurde, comme nous le verrons, par le Conseil des droits de l'homme de l'ONU), à l'instar de la notion de «sensibilités blessées », de façon à réintroduire, pour ainsi dire par la bande, la notion de blasphème.

Il y a eu nombre de débats sur l'extension de la notion de diffamation aux groupes. À l'origine, la diffamation est individuelle: un individu précis est fallacieusement accusé de telle ou telle turpitude par un autre individu qui veut lui donner «mauvaise réputation» de façon, par exemple, à affaiblir sa position dans la société. Dans un arrêt resté d'ailleurs isolée ${ }^{21}$, la Cour suprême des États-Unis a effectivement élargi le champ d'application de la diffamation en l'utilisant à l'égard de groupes et non plus seulement d'individus. Plus exactement, elle a appliqué la notion à des individus diffamés en tant que membres d'un groupe. Il s'agissait, notons-le bien, de propos brutalement racistes tenus par un suprématiste blanc. Une loi de l'Illinois, où l'affaire avait commencé, interdisait de tels propos - et cette loi fut contestée jusque devant la Cour suprême des États-Unis.

Deux grands juges, tous deux nommés par Roosevelt, s'opposèrent à ce sujet. Le juge Felix Frankfurter considérait qu'un des buts principaux des législations anti-diffamation consistait en ceci que la libre circulation de rumeurs négatives et mensongères à propos d'un individu empêchait qu'il soit traité selon ses mérites et ses capacités. Un employeur se dirait peut-être qu' 'il n'y a pas de fumée sans feu», alors qu'en réalité il en existe énormément - c'est d'ailleurs l'essence même de la diffamation. Frankfurter poursuivait son raisonnement en assimilant les propos racistes à la diffusion de préjugés et de stéréotypes à l'égard cette fois d'individus en tant que membres d'un groupe visé (ici les Africains-Américains). De tels «discours» racistes empêchaient que les Noirs soient traités selon leurs mérites et compétences:

21. Beauharnais v. Illinois, 343 U.S. 250 (1952). 
l'employeur apprécierait le candidat en fonction de ses préjugés (il appartient à un groupe à propos duquel les stéréotypes négatifs sont omniprésents) et non des qualités de ce dernier. Le juge Frankfurter en tirait comme conclusion que le racisme avait un effet similaire à la diffamation individuelle: il créait un contexte dans lequel un individu serait apprécié en fonction de préjugés ( «c'est un voleur»; "c'est un Noir et les Noirs sont des voleurs») et non de ses qualités propres.

Le juge Hugo Black, pour sa part, considérait que le droit de la diffamation devait rester confiné dans des limites strictes de façon à ne pas devenir dangereux pour la liberté d'expression. L'étendre à l'appartenance à un groupe discrédité par des propos racistes n'était pas souhaitable. Frankfurter l'emporta, mais Black, minoritaire, affirma: «Encore une victoire comme celle-là, et nous sommes fichus.» Une victoire à la Pyrrhus, en quelque sorte.

La Cour suprême des États-Unis n'a pas persévéré dans cette direction et a vite abandonné la notion de «diffamation collective». Mais c'est une notion utilisée par certains tribunaux européens et dans des contextes de débats politiques et idéologiques. Il faut bien savoir de quoi l'on parle pour éviter les effets potentiellement pervers de l'argument. Dans l'affaire Beauharnais, il s'agissait de racisme pur et simple, la religion n'avait rien à y faire. Pourtant, Frankfurter parle de groupes raciaux et religieux, créant par là même une difficulté à laquelle seront confrontés tous ceux qui feront usage (parfois immodérément) de la notion. Il y a en effet une différence importante entre la «race» et la religion. L'une constitue une assignation liée à des traits prétendument biologiques, c'est-à-dire inchangeables par les membres du groupe concerné. On est Noir, on ne devient pas Blanc par décret, et bien sûr vice versa. L'autre constitue un engagement spirituel, un choix auquel l'individu, dans une société libre, peut changer ce qu'il veut, jusqu'à l'abandonner et prendre une direction radicalement différente.

On peut donc comprendre l'argument de Frankfurter dans un contexte de propos racistes: quand on parle des Noirs, c'est de tel ou tel individu ayant la couleur en question qu'il s'agit, et c'est pour le coup que la qualification lui «colle à la peau». On peut effectivement parler avec une certaine cohérence de «diffamation collective» s'il est question de propos qui ont pour conséquence délétère qu'un individu sera traité en fonction de préjugés liés à son appartenance (dont il lui est impossible de se détacher), à l'instar de l'individu victime d'attaques verbales diffamatoires portant sur «lui seul» (diffamation individuelle). La Cour européenne des droits de l'homme a donc eu raison de parler de diffamation (collective) à propos du racisme, et même du négationnisme: 
« Il n'est pas non plus douteux que l'ingérence poursuivait un but légitime, à savoir la "protection de la réputation ou des droits d'autrui" 22. »

« [...] il ne fait aucun doute que contester la réalité de faits historiques clairement établis, tels que l'Holocauste, ne relève pas d'un travail de recherche historique s'apparentant à une quête de la vérité. Une telle démarche a en fait pour objectif de réhabiliter le régime national-socialiste et, par voie de conséquence, d'accuser de falsification de l'histoire les victimes elles-mêmes. La contestation de crimes contre l'humanité apparaît donc comme l'une des formes les plus aiguës de diffamation raciale envers les juifs et d'incitation à la haine à leur égard ${ }^{23}$.»

Si on peut donc parler légitimement de diffamation collective à propos d'individus appartenant à un groupe qui est l'objet de stéréotypes et d'expressions racistes, il faut absolument éviter un élargissement exagéré de la notion. Quand on l'étend aux groupes religieux, et non «raciaux» au sens strict du terme comme dans les affaires Beauharnais et Jersild, on entre dans un domaine où le choix compte, c'est-à-dire où l'individu peut se détacher de son appartenance, la réinterpréter, la récuser, changer de confession, devenir athée, etc. Certes, considérer l'appartenance religieuse comme un choix personnel contredit la réalité la plus commune, à savoir le fait que la toute grande majorité des personnes religieuses dans le monde adoptent la religion de leurs parents et restent donc toute leur vie dans leur groupe « de naissance». Pour beaucoup d'entre elles, leur identité religieuse fait corps avec ce qu'elles sont, presque autant que la couleur de leur peau. Mais il faut aussi envisager la question sous un autre angle: même si peu d'individus dans de nombreux pays le font (ils n'en ont d'ailleurs souvent pas le droit), il reste que le choix, la prise de distance, le changement sont toujours par principe possibles. En soi, la religion ne «colle» pas à l'individu comme la couleur de la peau (et les traits «raciaux» que les racistes voient « derrière»).

Cette différence a des conséquences importantes pour le problème de liberté d'expression qui nous occupe. En effet, le blasphème compris comme «diffamation collective» des membres d'une religion donnée est parfois (de façon très «périlleuse») considéré comme relevant des propos racistes. On dira: «Si vous dites des choses tellement négatives sur la croyance professée

22. CEDH, Gde ch., 23 sept. 1994, n 15890/89, Jersild c. Danemark, § 14. En question étaient des propos racistes tenus par des skinheads à Copenhague. Ces propos étaient odieux et de caractère général à l'égard des Noirs, assimilés à des animaux. Il s'agissait donc bien de diffamation collective.

23. CEDH, déc. 24 juin 2003, no 65831/01, Garaudy c. France. Il s'agit d'une décision d'irrecevabilité dans une affaire portant sur le négationnisme [souligné par nous]. 
par un groupe d'individus, si vous n'hésitez pas à vous en moquer, parfois cruellement, c'est que vous ne les aimez pas; pis, vous témoignez à leur égard d'une peur irrationnelle (phobie) qui vous mène à les haïr - bref vous êtes un raciste.»

On assisterait ici à un nouveau déplacement, prolongeant en quelque sorte celui que nous avions pointé plus haut. Dans un premier temps, on passait de l'offense à Dieu à l'offense ("gratuite») aux croyants. On restait donc dans le domaine religieux. Dans ce second temps, on passerait de l'accusation d'attaque contre les «sensibilités» religieuses à l'accusation de racisme - ce qui pourrait entraîner des conséquences redoutables pour la liberté d'expression.

Quand le problème était formulé en termes de blasphème, il y avait, du point de vue de la philosophie des droits de l'homme, une victime clairement identifiée, à savoir l'individu ou l'association dont le droit à la liberté d'expression était violé au nom de la défense de la religion (de Dieu). Le «bourreau » apparaissait également sans ambiguiité: c'était l'État insuffisamment laïcisé, continuant à se comporter en gardien de la religion. Quand la problématique fut traduite dans le langage des droits de l'homme, deux libertés se firent face: on ne savait pas très bien de quel côté se situait la «victime», la relation étant devenue pour ainsi dire horizontale. Était-ce la liberté d'expression, violée parce qu'on avait interdit des expressions heurtant, choquant, inquiétant, pourtant en théorie permises? Ou était-ce la liberté religieuse dans la mesure où on pouvait qualifier les propos incriminés de gratuitement offensants (non permis) ? Nous nous trouvions dans une grande insécurité juridique, et nous avons vu la Cour européenne des droits de l'homme trancher dans le sens de la liberté religieuse, la Cour suprême des États-Unis décider dans l'autre sens (ne faisant pas la distinction nébuleuse entre ce qui choque «simplement» et ce qui offense gratuitement). Maintenant, quand l'expression est qualifiée de raciste («islamophobe», «christianophobe», etc.), c'est celui qui exerce son droit à la liberté d'expression qui se trouve en position de «bourreau» (les propos racistes sont immoraux, et illégaux dans de nombreux pays). La situation a été complètement inversée.

Si l'on applique alors la notion de diffamation collective à ce supposé racisme-là, il s'agira à nouveau de brider la liberté d'expression au nom d'une valeur reconnue dans le contexte de la démocratie libérale: le droit à la réputation. Quelle leçon en tirer? Qu'il est extrêmement dangereux d'élargir le champ d'application de la notion de diffamation collective aux groupes religieux, dans la mesure où une telle extension du concept risque 
de «geler» la liberté d'expression en transformant la critique légitime de la religion, qualifiée pour l'occasion d'offense gratuite, en discours raciste. Et certes, toute la difficulté réside dans le fait que les groupes religieux peuvent faire l'objet d'attaques racistes, puisque la définition de l'entité stigmatisée dépend des fantasmes et de l'imagination débridée des racistes eux-mêmes. Le danger consisterait à taxer de diffamation collective (group libel) toute critique un peu «forte» (satirique, caricaturale, etc.) d'une religion.

Il faut toujours se rappeler que les religions relèvent non seulement de la spiritualité et d'une libre adhésion (ou non-adhésion) dans les sociétés laiques, mais qu'elles ont aussi parfois la redoutable tendance à faire prévaloir la loi de Dieu sur celle des hommes (dans les religions monothéistes, la supériorité ontologique du Créateur sur sa créature). Une bonne dose d'impertinence est nécessaire pour éviter les dérives. Il ne faudrait pas que cette dernière soit entravée par des accusations tous azimuts de "racisme " antireligieux, quitte à banaliser le racisme réel. Nous en avons vu un exemple frappant lors de l'affaire Giniewski, quand cet historien français avait été condamné pour avoir sévèrement critiqué le christianisme et «l'enseignement du mépris ${ }^{24}$ » dont les chrétiens s'étaient rendus coupables au cours de l'histoire vis-à-vis des juifs, jusqu'à la position très ambiguë prise par le pape Pie XII à propos de la Shoah. Il fut jugé et condamné pour «diffamation raciale $^{25}$ » et ne dut, si l'on peut dire, son «salut» qu'à la Cour européenne des droits de l'homme, qui condamna la France pour avoir violé l'article 10 de la Convention européenne des droits de l'homme garantissant la liberté d'expression ${ }^{26}$.

Mais nous ne sommes pas seulement confrontés aujourd'hui au double élargissement du champ d'application de la diffamation dont nous avons parlé: de la diffamation individuelle à la diffamation d'individus pour cause d'appartenance à un groupe victime de préjugés racistes, et de cette diffamation collective à la diffamation collective pour raisons religieuses. En effet, jusqu'ici (sauf dans le cas de l'affaire Giniewski où une communauté

24. V. J. IsAac, L'enseignement du mépris, Paris, Fasquelle, 1962.

25. La loi française de 1881 sur la liberté de la presse a été modifiée par la loi Pleven du $1^{\text {er }}$ juillet 1972 pour renforcer les instruments de la lutte contre le racisme: «Art. 32. [...] La diffamation commise envers les particuliers par l'un des moyens énoncés en l'article 23 sera punie d'une amende de 12000 euros. La diffamation commise par les mêmes moyens envers une personne ou un groupe de personnes à raison de leur origine ou de leur appartenance ou de leur non-appartenance à une ethnie, une nation, une race ou une religion déterminée sera punie...» [souligné par nous].

26. CEDH, 30 janv. 2006, no 64016/00, Giniewski c. France. 
chrétienne potentiellement indéfinie était dite «diffamée»), il s'agissait d'individus attaqués parce que membres d'un groupe, d'abord «racial», puis - extension très périlleuse - religieux. Le Conseil des droits de l'homme de l'ONU a franchi de nouvelles limites en votant durant plusieurs années des motions relatives à la diffamation des religions (la notion a été abandonnée en 2011). Ici, on en revenait à la conception première du blasphème (attaques contre la religion, contre Dieu), tout en conservant le vocabulaire de la diffamation, donc du droit à la réputation, donc des «droits de l'homme». Mais il ne s'agissait plus d'individus se disant, à tort ou à raison, attaqués en raison de leurs convictions religieuses: il était question «des religions». Le Conseil des droits de l'homme, traitant tout à fait légitimement du racisme (c'était l'objet de ces réunions) comme de l'un des «adversaires» majeurs des droits de l'homme, en venait à vouloir protéger «les religions» de la « diffamation». Ici, la dérive est radicale, la notion d'atteinte à la réputation perd tout sens, la corruption du langage (et donc de la pensée) des droits de l'homme est à son $\mathrm{acmé}^{27}$.

\section{4. «DISCOURS DE HAINE »}

Une autre façon de réprimer le blasphème au nom même des droits de l'homme et de la démocratie libérale consiste à le rattacher à la notion très ambiguë de hate speech. "Discours de haine»: tenons-nous-en ici à une interprétation stricte en termes d'incitation. L'incitation à la haine est souvent associée à l'incitation à la violence. Elle implique alors l'imminence d'un passage possible à l'acte. John Stuart Mill donne, dans On liberty (1859), l'exemple d'un vendeur de blé stockant sa marchandise. Supposons qu'un journaliste le traite d'affameur du peuple dans un journal, et l'accuse de créer une rareté artificielle pour faire monter les prix, risquant par là même d'engendrer une famine ${ }^{28}$. La qualification est dure, peut-être injuste, mais

27. Durant des années, l'Organisation de la coopération islamique (OCI) avait tenté de promouvoir l'inscription de la diffamation des religions comme une nouvelle forme de racisme, contre l'avis, notamment, des pays occidentaux. Déjà en 2001, l'ancienne Commission des droits de l'homme avait adopté une résolution en ce sens. En 2005, l'Assemblée générale de l'ONU adoptait également une résolution (non contraignante) contre la diffamation des religions. En décembre 2011, cette même Assemblée générale abandonnait le concept de "diffamation des religions». V. https://www.humanrights. $\mathrm{ch} / \mathrm{fr} /$ droits-humains-internationaux/nouvelles/divers-organes-de-lonu/diffamation-religions-lonu-abandonne-concept [consulté le 23 juin 2020].

28. V. J. S. MiLl, On Liberty, chap. III, «On individuality, as one of the elements of wellbeing», London, Penguin Books, 1984 [1859], p. 119. 
elle fait selon Mill partie du débat public. Supposons maintenant que le même discours soit prononcé mot à mot par un leader syndical devant la maison du vendeur de blé face à une foule excitée et prête à en découdre. On pourrait dire, dans la terminologie moderne (que Mill n'utilise pas) que le leader incite à la haine et rend la violence imminente et probable.

La Cour suprême des États-Unis a repris cette conception de la liberté d'expression dans son arrêt Brandenburg $v$. Ohio ${ }^{29}$. On notera que la justification de la répression du discours de haine ne dépend pas tellement de son contenu (la «haine»- s'il faut utiliser le terme - est déjà présente dans le discours du journaliste), mais du contexte dans lequel il est prononcé. Tant qu'il existe une certaine distance entre le discours et l'action qu'il pourrait susciter, on parlera d'advocacy, mais quand le lien entre l'expression et l'action violente se révélera trop «court», on considérera qu'il s'agit d'incitement. Défense sur le plan des idées (même provocantes ou choquantes) d'une part, incitation proprement dite d'autre part.

Cette distinction relativement claire (si on laisse de côté les différents cas limites - hard cases) est loin d'être toujours respectée. Elle ne l'a d'abord pendant longtemps pas été par la Cour suprême elle-même, qui a durant toute une période réprimé la simple défense (advocacy) d'idées communistes, liées au renversement violent du pouvoir de la «bourgeoisie» ${ }^{30}$. Elle est souvent beaucoup moins strictement appliquée en Europe - sans mentionner les régimes autoritaires dans lesquels, à la limite, toute critique du pouvoir peut être traitée comme une incitation à la violence. D'ailleurs, la thèse de Mill peut être contestée en tant que telle: est-il bien raisonnable d'attendre l'imminence du danger pour agir? Ne faut-il pas prévenir l'acte de haine et la violence en amont, quand il en est encore temps? N'existe-t-il pas une sorte de pente glissante menant du discours à l'action? Les législations européennes criminalisant le discours raciste vont dans ce sens quand elles ne réclament pas, pour que le discours ne soit plus protégé, l'imminence d'une action violente.

Mais encore une fois, si on applique une théorie plus souple que celle de Mill à la question du discours blasphématoire, on risque de condamner

29. «Freedoms of speech and press do not permit a State to forbid advocacy of the use of force or of law violation except where such advocacy is directed to inciting or producing imminent lawless action and is likely to incite or produce such action. »: Brandenburg v. Ohio, 395 U.S. 444 (1969) [souligné par nous].

30. V. G. HaARscher, La Cour suprême des États-Unis. Les droits de l'homme en question, Bruxelles, Académie royale de Belgique, 2014, p. 93-131. 
comme discours de haine toute critique un peu vigoureuse de la religion, des personnages sacrés et des Églises. Le refus de la haine et de la violence constitue une valeur cardinale des démocraties libérales, mais, à l'instar du respect des sensibilités et du refus de la diffamation, les lettres de crédit « libérales » de l'argument risquent de faire perdre de vue son caractère potentiellement liberticide.

\section{CONCLUSION PROVISOIRE}

Ce que j'ai tenté trop succinctement de montrer dans cet article, c'est à quel point aujourd'hui - la question du blasphème ne constitue à cet égard qu'un exemple particulièrement parlant - les arguments au nom desquels une pression de conformité s'exerce sur la liberté d'expression, en particulier quand il s'agit de religion, ont changé. Ce qui reste de législations anti-blasphème dans certains pays européens possède au moins le mérite de la clarté: il s'agit pourrait-on dire d'une attaque frontale contre la liberté d'expression au nom d'une valeur jugée supérieure. Certes, l'attaque par «ricochet» contre les croyants se trouve parfois mentionnée dans ces lois, mais le cœur même de l'infraction concerne, comme l'énonce l'article 188 du Code pénal autrichien, le «dénigrement de doctrines religieuses ${ }^{31}$ ».

De nombreux pays, comme la France et la Belgique, ne possèdent pas de législation anti-blasphème, mais le même résultat (l'interdiction des propos blasphématoires) peut être atteint par d'autres voies. La qualification de «blasphème» ne concorde pas avec la nature des sociétés pluralistes, et la Cour suprême des États-Unis a agi sagement (depuis 195232) en refusant d'intervenir, au nom de la séparation des Églises et de l'État, dans de telles controverses. Mais - et c'était l'objet du présent article - d'autres qualifications sont souvent utilisées en Europe dans des législations, des décisions de justice, des controverses idéologico-politiques (également aux États-Unis, particulièrement sur les campus universitaires pour ces dernières), qui ont pour caractéristique, quant à elles, de concorder avec les valeurs de la démocratie libérale. Qui ne considérerait pas comme un progrès de la «civilisation des mœurs» (Norbert Elias), le refus d'offenser gratuitement autrui, ce qui témoignerait d'un manque de respect, voire d'une atteinte à sa «dignité»? Qui ne considérerait pas qu'une société dans laquelle tout un chacun pourrait,

31. Arrêt Otto-Preminger-Institut c. Autriche, précit., \& 11.

32. Joseph Burstyn, Inc. v. Wilson, 343 U.S. 495 (1952). 
sans en assumer la responsabilité, répandre les rumeurs mensongères à sa discrétion, deviendrait totalement invivable? Qui ne dénoncerait pas les appels à la haine, à la violence et au meurtre si présents aujourd'hui sur les réseaux sociaux?

Mais il ne faut pas se tromper d'adversaire. Dans le combat pour la dignité, contre le racisme et les discours parfois ultraviolents, la libre critique - y compris la satire et la caricature - de la religion risque de se trouver emportée par un courant non maîtrisé de correction politique (political correctness). C'est de cette question que je me suis préoccupé: il n'y a pas d'offense gratuite quand l'expression litigieuse concerne un public volontaire et que la bonne manière de ne pas se trouver heurté dans ses sensibilités consiste tout simplement à ne pas aller voir le spectacle ou à ne pas ouvrir le livre (voire à ne pas consulter le site Internet en cause). L'impertinence «voltairienne» à l'égard des autorités constitue la condition sine qua non d'une action démocratique saine et vive. Il n'existe aucune raison pour que les autorités religieuses soient immunisées par rapport à une telle prise de distance critique. Il faut patiemment déconstruire (j'ai tenté de commencer à le faire ici) les différents arguments - une rhétorique parfois perverse - par lesquels l'impertinence à l'égard de la religion (qui n'exclut nullement le respect de la personne des croyants) se trouve qualifiée d'offense gratuite, de diffamation collective ou d'incitation à la haine (des pratiquants). Nous avons encore beaucoup de pain sur la planche. 